\title{
ANALYSIS ON THE HEISENBERG MANIFOLD
}

BY

\author{
RICHARD TOLIMIERI
}

\begin{abstract}
A study of the function theory on the Heisenberg manifold in terms of theta functions. Subject to an explicit error, a $C^{\infty}$-function is written as an infinite sum, with theta functions of different degrees and characteristics playing the same role as exponentials do in the abelian theory.
\end{abstract}

1. Introduction. The motivation for the viewpoint of this work requires a brief discussion of the historical evolution of some of the ideas of Fourier analysis on the torus $\Pi=R / Z$. Probably, the most universally known aspect of this theory concerns the space $\mathfrak{L}^{2}(\Pi)$ of square integrable functions. This theory centers about the characters $e^{2 \pi i n x}, n \in Z$, of $\Pi$ (identifying $\Pi$ with the interval $[0,1]$ in the usual manner). The characters determine an orthonormal basis of the Hilbert space $\mathscr{L}^{2}(\Pi)$ and associating to each $f \in \mathcal{L}^{2}(\Pi)$ its corresponding Fourier series $\Sigma \hat{f}(n) e^{2 \pi i n x}$, where $\hat{f}(n)=\int_{0}^{1} f(t) e^{-2 \pi i n t} d t$, the Riesz-Fischer theorem establishes the identification between the space $\mathfrak{L}^{2}(\Pi)$ and the space of trigonometric series $\Sigma a_{n} e^{2 \pi i n x}$ where $\Sigma\left|a_{n}\right|^{2}<\infty$. In general, the Fourier series of a function in $\mathfrak{L}^{2}(\Pi)$ converges in the $\mathfrak{L}^{2}$-norm to the function. Additional assumptions on the function in question are necessary if more is required. Our primary interest is the classical assertion that the Fourier series of a $\mathrm{C}^{\mathrm{l}}$-function on $\Pi$ converges absolutely and uniformly to the function.

Group representation theory serves to reinterpret the classical theory and, equally importantly, determines new questions. Returning to our example, if we define the translation representation $T$ of $R$ on $\mathcal{L}^{2}(\Pi)$ by the formula

$$
\left(T_{y} f\right)\left(e^{2 \pi i x}\right)=f\left(e^{2 \pi i(x+y)}\right), \quad f \in \mathcal{L}^{2}(\Pi) \text { and } x, y \in R,
$$

it is easy to verify that $T$ is a continuous representation for which the characters constitute a complete system of eigenvectors. In this light the naturality of the characters as a cornerstone in this theory is further illuminated. In this and other analogous settings one pursues information about important $T$-invariant subspaces.

Received by the editors January 31, 1975 and, in revised form, December 2, 1975. AMS (MOS) subject classifications (1970). Primary 22E25; Secondary $22 \mathrm{E} 30$.

Key words and phrases. Theta functions, Heisenberg group. 
The prejudice of this work is that for the most part current interest about analysis on groups has emphasized the spirit native to representation theory, neglecting problems born from a classical perspective. Two works of $J$. Brezin [3] and [4] are notable exceptions. In this work we hope to justify a classical approach at least to the extent of showing that something of interest can be said about the function theory of nil-manifolds using this approach. Indispensable to our development is the theory of theta functions and their interplay with the representation theory. Below we indicate the necessary highlights of this marriage but for a more detailed description one may refer to [2].

Specifically, the nil-manifold under investigation is the 3-dimensional Heisenberg manifold described below. Compared to the situation confronted on the torus, the function theory of this manifold thirsts primarily from a lack of a convenient basis which would play the variety of roles analogous to those of the characters. However, as we shall see, for certain kinds of problems explicit use of theta functions enables one to construct an appropriate basis.

The Heisenberg group $N_{3}$ is defined as follows

$$
N_{3}=\left\{\left(\begin{array}{lll}
1 & y & z \\
0 & 1 & x \\
0 & 0 & 1
\end{array}\right): x, y, z \in R\right\}
$$

Let $\Gamma$ be the integer matrices in $N_{3}$. The Heisenberg manifold is the homogeneous space of right cosets $\Gamma \backslash N_{3}=\left\{\Gamma_{n} \mid n \in N_{3}\right\}$. Clearly, $\Gamma \backslash N_{3}$ is compact. Denoting by $(x, y, z)$ the matrix

$$
\left(\begin{array}{lll}
1 & y & z \\
0 & 1 & x \\
0 & 0 & 1
\end{array}\right)
$$

the multiplication rule becomes $(x, y, z)\left(x^{\prime}, y^{\prime}, z^{\prime}\right)=\left(x+x^{\prime}, y+y^{\prime}, z+z^{\prime}\right.$ $\left.+y x^{\prime}\right)$.

It is known that a unique probability measure can be defined on $\Gamma \backslash N_{3}$, invariant under right translations. This measure will be assumed in all that follows. Let $\mathcal{L}^{2}\left(\Gamma \backslash N_{3}\right)$ be the Hilbert space of square integrable functions on $\Gamma \backslash N_{3}$. The right quasi-regular representation of $N_{3}$ on $\mathcal{L}^{2}\left(\Gamma \backslash N_{3}\right)$ is defined by the formula

$$
\left(\Re_{x} F\right)(y)=F(y x), \quad x, y \in N_{3}, F \in \mathcal{L}^{2}\left(\Gamma \backslash N_{3}\right) .
$$

The unitary representation $\Re$ has been studied in detail in [1] and reconsidered from a slightly different vantage in [2]. For our immediate purposes it is sufficient to recall the following facts. The space $\mathfrak{L}^{2}\left(\Gamma \backslash N_{3}\right)$ decomposes into an orthogonal direct sum of $\mathcal{R}$-invariant spaces 


$$
\mathcal{L}^{2}\left(\Gamma \backslash N_{3}\right)=\sum_{n \in Z} \bigoplus H_{n}
$$

where $H_{n}$ consists of the space of all functions in $\mathcal{L}^{2}\left(\Gamma \backslash N_{3}\right)$ satisfying the following condition

$$
\Re_{(0,0, z)} F=e^{2 \pi i n z} \cdot F, \quad F \in \mathcal{L}^{2}\left(\Gamma \backslash N_{3}\right), z \in R .
$$

The multiplicity of the representation $\Re$ on $H_{n}$ is $|n|$ when $n \neq 0$ and in particular $H_{1}, H_{-1}$ are $R$-invariant and irreducible subspaces. The space $H_{1}$ is crucial in the analysis of $\mathfrak{L}^{2}\left(\Gamma \backslash N_{3}\right)$ and we initiate our investigations by recalling the relationship between $H_{1}$ and the theta functions of order 1. Let

$$
\theta\left[\begin{array}{l}
a \\
b
\end{array}\right](z, \tau)=\sum_{n \in Z} e^{\pi i \tau(n+a / 2)^{2}} e^{2 \pi i(n+a / 2)(z+b / 2)}
$$

where $a, b \in R, z=x+i y \in \mathbf{C}$ and $\operatorname{im}(\tau)>0$. The usual complex analysis arguments show that $\theta\left[{ }_{b}^{a}\right](z, \tau)$ is an entire function in $z$ for each choice of $a, b \in R$ and $\operatorname{im}(\tau)>0$. The function $\theta\left[\begin{array}{c}a \\ b\end{array}\right](z, \tau)$ is called the Jacobi theta function of argument $z$, period $\tau$ and characteristic $\left[\begin{array}{l}a \\ b\end{array}\right]$. Associated to $\theta\left[\begin{array}{l}a \\ b\end{array}\right](z, \tau)$ we define the function $\psi\left[\begin{array}{l}a \\ b\end{array}\right]$ on $N_{3}$ by the formula

$$
\psi\left[\begin{array}{l}
a \\
b
\end{array}\right](x, y, z, \tau)=e^{2 \pi i z} e^{2 \pi i(b y / 2-a x / 2)} e^{\pi i \tau y^{2}} \theta\left[\begin{array}{l}
a \\
b
\end{array}\right](x+\tau y, \tau) .
$$

Using the uniform convergence (on compact subsets) with respect to $z$ of the series implicit on the right we can rewrite $\psi\left[\begin{array}{l}a \\ b\end{array}\right]$ as follows

$$
\psi\left[\begin{array}{l}
a \\
b
\end{array}\right](x, y, z, \tau)=e^{2 \pi i a b / 4} e^{2 \pi i z} \sum_{n} e^{\pi i \tau(y+n+a / 2)^{2}} e^{2 \pi i b(n+y) / 2} e^{2 \pi i n x} .
$$

It is a simple exercise to show that $\psi\left[{ }_{b}^{a}\right](x, y, z, \tau) \in H_{1}$, for each choice $a, b \in R, \operatorname{im}(\tau)>0$. This procedure gives a collection of special functions in $H_{1}$ relative to which one can attempt to structure $H_{1}$. We shall single out two of these functions for special consideration. Set

$$
\psi(x, y, z)=\psi\left[\begin{array}{l}
0 \\
0
\end{array}\right](x, y, z, i), \quad \psi^{*}(x, y, z)=\psi\left[\begin{array}{l}
1 \\
1
\end{array}\right](x, y, z, i) .
$$

Let $\chi_{n m}=e^{2 \pi i(n x+m y)}, n, m \in Z$. The role of the characters on $\Pi$ will be performed by the functions $\left\{\chi_{n m} \cdot \psi(x, y, z) ; n, m \in Z\right\}$. In [2, Theorem II.5], it is proved that these functions define an $\mathcal{L}^{2}$-basis of $H_{1}$ in the sense that the closure of the linear span of these functions is $H_{1}$ and these functions are minimal with respect to this property. Observe that they are not orthonormal. The main question that comes to mind is whether some natural condition on a function $F \in H_{1}$ implies the representation of $F$ as a uniformly converging series in our $\mathcal{L}^{2}$-basis. Although this is not exactly the case we have a measure of error. The tool of this study, which occurs in $\$ 5$, is provided by our main theorem in $\$ 4$ which we are now in a position to state. Let $\bigodot_{1}$ be the er -functions in $H_{1}$ and $\gamma_{1}(x, y)=\sin x-i \sin y$. 
MAIN Theorem. For $F \in \mathcal{C}_{1}^{n}, n \geqslant 1$, there exists a unique function $p \in$ $e^{n-1}\left(\pi^{2}\right)$ and a unique polynomial $h$ in $\gamma_{1}$ of degree $n$, i.e. $h=\sum_{e=0}^{n} a_{e} \gamma_{1}^{e}(x, y)$ such that $F=p \psi+h \psi_{-i}^{*}$ where $\psi_{-i}^{*}=\beta_{-i} \psi^{*}, \beta_{-i}=1-e^{2 \pi i x}+e^{2 \pi i(x-y)}-$ $e^{-2 \pi i y}$.

The artificiality of the form $\beta_{-i}$ is cleared by the results of $\S 3$.

In $\S 6$ we will extend our main theorem to the other $H_{n}$ 's, $n \neq 0$.

Also we prove (see Theorem 2.3) that functions in $H_{1}$ are controlled in a manner which hopefully will be made clear by the nature of their zeroes. In fact each function which is continuous and in $H_{n}, n \neq 0$, vanishes at some point [2, Theorem II.2]. The exact statement of our result is contained in the following.

THEOREM. If $F \in \mathcal{C}_{1}^{1}$, then $1 / \bar{F} \notin \mathcal{L}^{2}\left(\Gamma \backslash N_{3}\right)$.

The final preliminary notion corresponds essentially to the notion of the Fourier transform on $\mathcal{L}^{2}(R)$. We shall be slightly more general than necessary in our formulation. Let $S L_{2}(Z)$ be the group of all $2 \times 2$ integer matrices having determinant 1 . This group acts as a group of automorphisms of $\mathrm{N}_{3}$ according to

$$
\left(\begin{array}{ll}
a & b \\
c & d
\end{array}\right)(x, y, z)=\left(c y+d x, a y+b x, z+\frac{1}{2}\left(b d x^{2}+2 b c x y+a c y^{2}\right)\right),
$$

where $\left(\begin{array}{ll}a & b \\ c & d\end{array}\right) \in S L_{2}(Z)$ and $(x, y, z) \in N_{3}$. Letting $L$ be the subgroup of $S L_{2}(Z)$ defined by requiring $b d \equiv a c \equiv 0 \bmod 2$, it is easy to see that $L$ takes $\Gamma$ onto itself and acts on $H_{1}$ as a group of unitary operators by

$$
\sigma(F)(x, y, z)=F(\sigma(x, y, z)), \quad \sigma \in L, F \in H_{1} .
$$

The notion of the Fourier transform enters the picture in the following manner. It is a fact (see [5]) that the space $H_{1}$ corresponds to functions on $N_{3}$ of the form $e^{2 \pi i z} \sum_{n} f(n+y) e^{2 \pi i n x}$ where $f \in L^{2}(R)$. Put $\sigma=\left(\begin{array}{ll}0 & 1 \\ -1 & 0\end{array}\right)$. Then if $F=e^{2 \pi i z} \Sigma_{n} f(n+y) e^{2 \pi i n x}$, we have $\sigma(F)=e^{2 \pi i z} \Sigma_{n} \hat{f}(n+y) e^{2 \pi i n x}$ where $\hat{f}$ is the Fourier transform of $f$. Thus the unitary operator of $H_{1}$ determined by $\sigma$ gives a geometric interpretation of the Fourier transform. It will play an important role in this work as well.

2. Decomposition theorem. In this section we shall analyze the role which the "theta functions" $\psi$ and $\psi^{*}$ play in structuring the space $H_{1}$. It is simple to show that $H_{1}$ is a module over the ring $C_{0}=C\left(\Pi^{2}\right)$ of continuous functions on the two-dimensional torus $\Pi^{2}$, the operation of $C_{0}$ on $H_{1}$ being defined by function multiplication. Also given any two functions $F, G$ in $H_{1}$ the quotient $F / G$ is defined on $\Pi^{2}$ but may not be in $\bigodot_{0}$. Let $F$ be a function in $H_{1}$. Then $F \cdot \psi^{-1}$ can be considered as a function on $\Pi^{2}$. This observation implies that each $F \in H_{1}$ can be written uniquely in the form $F=p \cdot \psi$ where $p$ is defined 
on $\Pi^{2}$. Setting $\mathscr{F}_{1}\left(\Pi^{2}\right)$ equal to the space of periodic functions on $\Pi^{2}$ of the form $F / \psi, F \in H_{1}$ our observation becomes simply the assertion that $H_{1}=$ $\mathscr{F}_{1}\left(\Pi^{2}\right) \cdot \psi$. The problem with this decomposition of $H_{1}$ stems from the lack of control one has over the functions in $\mathscr{F}_{1}\left(\Pi^{2}\right)$. To see that this is the case we must consider the zeroes of functions in $H_{1}$ and in particular the zeroes of $\psi$. The proof of the following lemma appears in [2, Theorem II.2].

LEMMA 2.1. The function $\psi$ has as its zero set the points $\left(\frac{1}{2}, \frac{1}{2}, 0\right) \Gamma \cdot \mathcal{Z}$ where $\mathscr{Z}=\{(0,0, z): z \in R\}$.

Every continuous function $F \in H_{1}$ vanishes at some point.

The first statement of the lemma is just a translation of the classical result concerning the zero of the theta function $\theta\left[\begin{array}{l}0 \\ 0\end{array}\right](z, i)$ at $z=1 / 2+i / 2$ and the uniqueness of this zero in the unit square. Also it should be remarked that the zero of the entire function $\theta\left[{ }_{0}^{0}\right](z, i)$ is of order one. The second statement is remarkable in that the existence of a zero is shown to be independent of any analytic considerations. Also it implies that we cannot considerably improve the theory by taking quotients of functions in $H_{1}$ with denominators other than $\psi$. In fact, we can be more specific as to the nature of the difficulty.

The following proof was suggested by the referee.

THEOREM 2.3. If $F \in \mathcal{C}_{1}^{1}$, then $1 / \bar{F} \notin H_{1}$.

Proof. Let $F$ be $\mathcal{C}_{1}^{1}$, so $F(x, y, z)=e^{2 \pi i z} f(x, y)$. So $f$ is $\mathcal{C}^{1}$ also, and $|F| \equiv|f|$. Let $F$ have a zero at $\left(x_{0}, y_{0}, z_{0}\right)$, and let the partial derivatives of $f$ at $\left(x_{0}, y_{0}\right)$ be $\alpha$ and $\beta$ respectively. Then

$$
\left|F\left(x_{0}+h_{1}, y_{0}+h_{2}, z_{0}+h_{3}\right)\right| \approx\left|\alpha h_{1}+\beta h_{2}\right| \text {. }
$$

Hence the triple integral from $x_{0}$ to $x_{0}+S, y_{0}$ to $y_{0}+S$ and $z_{0}$ to $z_{0}+S$ of $|F|^{-2}$ is

$$
\begin{aligned}
\iiint|F|^{-2} d x d y d z & \approx S \int_{0}^{S} \int_{0}^{S}\left|\alpha h_{1}+\beta h_{2}\right|^{-2} d h_{1} d h_{2} \\
& \geqslant S \int_{0}^{S} \int_{0}^{S}\left(|\alpha| h_{1}+|\beta| h_{2}\right)^{-2} d h_{1} d h_{2}=\infty
\end{aligned}
$$

We do, however, have the following control over functions in $H_{1}$. For a proof see [2, Theorem II.4].

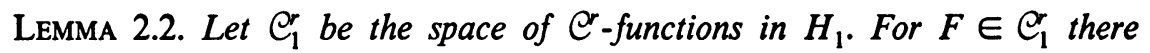
exists $p, q \in \mathcal{C}^{r}\left(\Pi^{2}\right)$ such that $F=p \psi+q \psi^{*}$.

It is important to observe that no mention of the uniqueness of $p, q$ has been made. In fact, a theorem of J. Brezin (see Theorem II.9 of [2]) states that $\mathcal{C}_{1}^{r}$ is a projective module over $\mathcal{C}^{r}(\Pi)$ and hence the nonuniqueness is inherent. 
3. The operator $\sigma=\left(\begin{array}{cc}0 & 1 \\ -1 & 0\end{array}\right)$. Set $\sigma=\left(\begin{array}{cc}0 & 1 \\ -1 & 0\end{array}\right)$ for the rest of the paper. The matrix $\sigma$ determines a unitary operator on $H_{1}$ as discussed in the introduction. The following notation will be convenient. For $p(x, y) \in \mathcal{C}\left(\Pi^{2}\right)$ denote by $p^{\sigma}$ the function in $\mathrm{C}\left(\Pi^{2}\right)$ defined by

$$
p^{\sigma}(x, y)=p(-y, x) \text {. }
$$

Set $T_{\sigma} F=\sigma(F), F \in H_{1}$, as defined on page 332. The operator $p \mapsto p^{\sigma}$ clearly has order four and hence decomposes $\mathcal{C}\left(\Pi^{2}\right)$ into the direct sum

$$
\bigodot\left(\Pi^{2}\right)=X_{1} \oplus X_{-1} \oplus X_{i} \oplus X_{-i}
$$

where $X_{\alpha}$ is the eigenvector space corresponding to the eigenvalue $\alpha=$ $\pm 1, \pm i$. The projection mappings $q_{\alpha}: \mathcal{C}\left(\Pi^{2}\right) \rightarrow X_{\alpha}$ are given by

$$
q_{\alpha}(p)=\frac{1}{4}\left(p+\alpha p^{-\sigma}+\alpha^{2} p^{-\sigma^{2}}+\alpha^{3} p^{-\sigma^{3}}\right) .
$$

Set $p_{\alpha}=q_{\alpha}(p)$. Analogously $T_{\sigma}$ has order four and we have a direct sum decomposition of $\mathcal{C}_{1}^{0}\left(\Gamma \backslash N_{3}\right)=\mathcal{C}_{1}$,

$$
e_{1}=Y_{1} \oplus Y_{-1} \oplus Y_{i} \oplus Y_{-i}
$$

where $Y_{\alpha}$ corresponds to the eigenvalues $\alpha= \pm 1, \pm i$ and the projection mappings $q_{\alpha}^{*}: \bigodot_{1} \rightarrow Y_{\alpha}$ are given by

$$
q_{\alpha}^{*}(F)=\frac{1}{4}\left(F+\alpha F^{-\alpha}+\alpha^{2} F^{-\alpha^{2}}+\alpha^{3} F^{-\sigma^{3}}\right) .
$$

Set $F_{\alpha}=q_{\alpha}^{*}(F)$.

The goal of this section is twofold. First we want to exhibit the existence of a decomposition $F=p \psi+q \psi^{*}$ in some sense compatible with the eigenvector space decomposition above. Finally we want to analyze and explicitly compute the projections of $\psi^{*}$ onto the different eigenvector spaces.

Lemma 3.1. For $F \in \mathcal{C}_{1} \cap Y_{\alpha}, r \geqslant 0$, and $\alpha= \pm 1, \pm i$ there exists $p, q \in$ er $\left(\Pi^{2}\right)$ such that

(i) $F=p \psi+q \psi^{*}$,

(ii) $p^{\sigma}=\alpha \cdot p$,

(iii) $q^{\sigma}(x, y)=-i \cdot \alpha \cdot e^{-2 \pi i x} q(x, y)$.

Proof. Recall from [2, Theorem III.6] that $\psi^{\sigma}=\psi$ and $\left(\psi^{*}\right)^{\circ}=i e^{2 \pi i x} \psi^{*}$. Let $F=p_{1} \psi+q_{1} \psi^{*}$ where $p_{1}, q_{1} \in \operatorname{er}^{r}\left(\Pi^{2}\right)$. Since $F \in Y_{\alpha}, F=q_{\alpha}^{*}(F)=p \psi$ $+q \psi^{*}$ where

$$
p=\frac{1}{4}\left(p_{1}+\alpha p_{1}^{-\sigma}+\alpha^{2} p_{1}^{-\sigma^{2}}+\alpha^{3} p_{1}^{-\sigma^{3}}\right)
$$

and

$$
q=\frac{1}{4}\left(q_{1}+\alpha i q_{1}^{-\sigma} e^{2 \pi i x}+\alpha^{2} i^{2} q_{1}^{\sigma^{-2}} e^{2 \pi i(x-y)}+\alpha^{3} i^{3} q_{1}^{-\sigma^{3}} e^{2 \pi i y}\right) .
$$

From $F^{\sigma}=\alpha F$ and $(p \psi)^{\sigma}=p^{\sigma} \psi=\alpha p \psi$ it follows that $\left(q \psi^{*}\right)^{\sigma}=\alpha q \psi^{*}$ and $q^{\sigma}=-i e^{-2 \pi i x} q$ whenever $\psi^{*} \neq 0$. The continuity of $q$ and the fact that $\psi^{*}$ 
vanishes only at the points $(0,0,0) \Gamma \mathscr{Z}$ implies (iii), and completes the proof of the lemma.

Lemma 3.1 can immediately be used to gain some information about the functions in the spaces $Y_{\alpha}$.

COROLlaRY 3.1.1. Every function in $Y_{1}$ vanishes at $\left(\frac{1}{2}, \frac{1}{2}, 0\right)$.

Corollary 3.1.2. Every function in $Y_{-1}$ vanishes at $(0,0,0)$ and $\left(\frac{1}{2}, \frac{1}{2}, 0\right)$.

Corollary 3.1.3. Every function in $Y_{i}$ vanishes at $(0,0,0),\left(\frac{1}{2}, 0,0\right)$, $\left(0, \frac{1}{2}, 0\right)$ and $\left(\frac{1}{2}, \frac{1}{2}, 0\right)$.

Corollary 3.1.4. Every function in $Y_{-i}$ vanishes at $(0,0,0),\left(\frac{1}{2}, 0,0\right)$ and $\left(0, \frac{1}{2}, 0\right)$.

Proof. We shall prove the first corollary and leave the proofs of the others to the reader. Choose $F \in Y_{1}$ and write $F=p \psi+q \psi^{*}$ as before (Lemma 3.1). Then $q\left(-\frac{1}{2}, \frac{1}{2}\right)=q\left(\frac{1}{2}, \frac{1}{2}\right)$ by periodicity and $q\left(-\frac{1}{2}, \frac{1}{2}\right)=-q\left(\frac{1}{2}, \frac{1}{2}\right)$ by the prvious lemma. Hence $q\left(\frac{1}{2}, \frac{1}{2}\right)=0$ and the vanishing of $\psi$ at $\left(\frac{1}{2}, \frac{1}{2}, 0\right)$ completes the proof.

Thus far except for $\psi \in Y_{1}$ we have no explicit examples of functions in $Y_{\alpha}, \alpha= \pm 1, \pm i$. The following lemma satisfies this need in a most useful manner for our later requirements. We have simply stated results since they are simple consequences of Lemma 3.1.

LEMMA 3.2. Put $\psi_{\alpha}^{*}=q_{\alpha}^{*}\left(\psi^{*}\right)$ the projection of $\psi^{*}$ into $Y_{\alpha}$. Then $\psi_{\alpha}^{*}=$ $\beta_{\alpha}(x, y) \cdot \psi^{*}$ where

(i) $\beta_{1}=1+i e^{2 \pi i x}-e^{2 \pi i(x-y)}-i e^{-2 \pi i y}$.

(ii) $\beta_{-1}=1-i e^{2 \pi i x}-e^{2 \pi i(x-y)}+i e^{-2 \pi i y}$.

(iii) $\beta_{i}=1+e^{2 \pi i x}+e^{2 \pi i(x-y)}+e^{-2 \pi i y}$.

(iv) $\beta_{-i}=1-e^{2 \pi i x}+e^{2 \pi i(x-y)}-e^{-2 \pi i y}$.

In [2, pp. 85-87] a study was made of the zero sets of $\psi_{\alpha}^{*}, \alpha= \pm 1, \pm i$ and we state without proof the conclusions of that analysis in the following lemma.

LEMMA 3.3. (i) The zeroes of $\beta_{1}$ occur at $(0,0)$ and $\left(\frac{1}{2}, \frac{1}{2}\right)$ (in the unit square) and the linear terms of the Taylor series at these points is given as follows:

at $(0,0),-2 \pi(1+i)(x-i y)$,

at $\left(\frac{1}{2}, \frac{1}{2}\right), 2 \pi(1-i)\left(\left(x-\frac{1}{2}\right)+i\left(y-\frac{1}{2}\right)\right)$.

(ii) The zeroes of $\beta_{-1}$ occur at $(0,0)$ and $\left(\frac{1}{2}, \frac{1}{2}\right)$ and the linear terms of the Taylor series at these points is given by

at $(0,0), 2 \pi(1-i)(x+i y)$, at $\left(\frac{1}{2}, \frac{1}{2}\right),-2 \pi(1+i)\left(\left(x-\frac{1}{2}\right) i\left(y-\frac{1}{2}\right)\right)$. 
(iii) The zeroes of $\beta_{i}$ occur on the lines $\left(\frac{1}{2}, y\right)$ and $\left(x, \frac{1}{2}\right)$ and the corresponding linear terms of the Taylor series are

$$
\begin{aligned}
& \text { at }\left(\frac{1}{2}, \frac{1}{2}\right), 0, \\
& \text { at }\left(\frac{1}{2}, y_{0}\right) \neq\left(\frac{1}{2}, \frac{1}{2}\right),-2 \pi i\left(1+e^{-2 \pi i y_{0}}\right)\left(x-\frac{1}{2}\right), \\
& \text { at }\left(x_{0}, \frac{1}{2}\right) \neq\left(\frac{1}{2}, \frac{1}{2}\right), 2 \pi i\left(1+e^{2 \pi i x_{0}}\right)\left(y-\frac{1}{2}\right) \text {. }
\end{aligned}
$$

(iv) The zeroes of $\beta_{-i}$ occur on the lines $(x, 0)$ and $(0, y)$ and the Taylor series at these points has linear terms

$$
\begin{aligned}
& \text { at }(0,0), 0, \\
& \text { at }\left(0, y_{0}\right) \neq(0,0),-2 \pi i\left(1-e^{-2 \pi i y_{0}}\right) x, \\
& \text { at }\left(x_{0}, 0\right) \neq(0,0), 2 \pi i\left(1-e^{2 \pi i x_{0}}\right) y \text {. }
\end{aligned}
$$

As a final comment about explicit functions in the spaces $Y_{\alpha}$ we note that Corollaries 3.1.1-3.1.3 are the best possible in the sense that in each of the eigenspaces $Y_{1}, Y_{-1}$ and $Y_{i}$ there are functions having the necessary zeroes as their zero set. For example $\psi$ has $\left(\frac{1}{2}, \frac{1}{2}, 0\right) \Gamma \mathscr{Z}$ as its zero set and lies in $Y_{1}, \psi_{-1}^{*}=\beta_{-1} \psi^{*}$ has $(0,0,0) \Gamma \mathscr{Z} \cup\left(\frac{1}{2}, \frac{1}{2}, 0\right) \Gamma \mathscr{Z}$ as its zero set and lies in $Y_{-1}$. Finally setting $\gamma(x, y)=\sin x+i \sin y$, we get $\gamma \psi \in Y_{i}$ and has the required zero set as its zero set. In $Y_{-i}$ all the author has been able to show is the existence of two functions $(\sin y+i \sin x) \psi$ and $\psi_{-i}^{*}$ in $Y_{-i}$ whose zero sets intersect to give the required zero set.

4. Main theorem. For a function $F \in H_{1}$ if we could write $F=p(x, y) \cdot \psi$ where $p$ were a smooth enough function on $\Pi^{2}\left(e^{2}\left(\Pi^{2}\right)\right.$ would do), then the Fourier series of $p(x, y)$ would converge absolutely to $p(x, y)$ and we would have a corresponding good representation for $F$. However, there seems no natural condition on $F$ which would insure a smooth enough $p$. The representation $F=p \psi+q \psi^{*}$ asserted in Lemma 3.1 does carry the smoothness of $F$ over into smoothness of $p$ and $q$; however, the nonuniqueness involved does not allow for the development of the usual kind of Fourier analysis. The main object of this section is to refine Lemma 3.1 in such a way that uniqueness is recovered.

Let $F \in \mathcal{C}_{1}$. Set $\gamma_{1}(x, y)=\sin x-i \sin y$. We shall spend the remainder of this section proving the following theorem.

THEOREM 4.1. For $F \in \mathcal{C}_{1}^{n}, n \geqslant 1$, there exists a unique $p \in \mathcal{C}^{n-1}\left(\Pi^{2}\right)$ and a unique polynomial $h$ in $\gamma_{1} \cdot h(x, y)=\sum_{l=0}^{n} a_{l} \gamma_{1}^{l}(x, y)$ such that $F=p \cdot \psi+h$. $\psi_{-i}^{*}$

The proof of this theorem will be broken up into a series of steps where we investigate what occurs in each of the eigenspaces and then put together into the global statement. Incidentally, this prodecure will refine Theorem 4.1 whenever we are dealing with functions belonging to the eigenspaces themselves. 
Let $F \in \mathcal{C}_{1}^{n} \cap Y_{1}, n>1$. We can write $F=p \psi+q \psi^{*}$ where $p, q \in$ $\operatorname{er}\left(\Pi^{2}\right)$ and satisfy the following functional equations.

$$
\begin{aligned}
& p(-y, x)=p(x, y), \\
& q(-y, x)=-i e^{-2 \pi i x} q(x, y) .
\end{aligned}
$$

It will be convenient to introduce the change of variables $z=x+i y, \bar{z}=$ $x-i y$. The functional equations become
$p(i z,-i \bar{z})=p(z, \bar{z})$,$$
q(i z,-i \bar{z})=-i e^{-\pi i(z+\bar{z})} q(z, \bar{z}) .
$$

We want to investigate the properties of the function $q \psi^{*} / \psi$. Clearly it is periodic and is $C^{r}$ except perhaps at $\left(\frac{1}{2}, \frac{1}{2}\right)$ where $\psi$ vanishes. Since $\psi$ equals $\left(x+\frac{1}{2}\right)+i\left(y+\frac{1}{2}\right)$ times a $\bigodot^{\infty}$-function not vanishing in some neighborhood of $\left(\frac{1}{2}, \frac{1}{2}\right)$ to study the smoothness of $q \psi^{*} / \psi$ at $\left(\frac{1}{2}, \frac{1}{2}\right)$ comes down to studying the smoothness of $q /\left(x+\frac{1}{2}\right)+\left(y+\frac{1}{2}\right) i$ at $\left(\frac{1}{2}, \frac{1}{2}\right)$. This is easily seen to be equivalent to studying the smoothness of $q^{*} / z$ where $q^{*}(x, y)=$ $q\left(x+\frac{1}{2}, y+\frac{1}{2}\right)$ at $(0,0)$ where $q^{*}$ satisfies the functional equation:

$$
q^{*}(i z,-i z)=i e^{-\pi i(z+\bar{z})} q^{*}(z, \bar{z}) .
$$

The following two lemmas give the necessary information.

LEMMA 4.1. For $q^{*} \in \mathcal{C}^{n}\left(\Pi^{2}\right), n \geqslant 1$, satisfying (4.3), we have

$$
\begin{aligned}
(-i)^{n} \frac{\partial^{n}}{\partial \bar{z}^{n}} q^{*}(i z, & -i \bar{z}) \\
& =i e^{-\pi i(z+\bar{z})} \sum_{j=0}^{n}\left(\begin{array}{c}
n \\
n-j
\end{array}\right)(-\pi i)^{j} \frac{\partial^{n-j}}{\partial \bar{z}^{n-j}} q(z, \bar{z}) .
\end{aligned}
$$

Pkoof. The case $n=0$ is given by (4.3). Suppose the lemma is true for $r=j<n$. Then differentiating (4.4) with $n$ replaced by $j$ gives

$$
\begin{aligned}
(-i)^{j+1} & \frac{\partial^{j+1}}{\partial \bar{z}^{j+1}} q^{*}(i z,-i \bar{z}) \\
= & i(-\pi i) e^{-\pi(z+\bar{z})} \sum_{l=0}^{j}\left(\begin{array}{c}
j \\
j-l
\end{array}\right)(-\pi i)^{l} \frac{\partial^{j-l}}{\partial \bar{z}^{j-l}} q(z, \bar{z}) \\
& +i e^{-\pi i(z+\bar{z})} \sum_{l=0}^{j}\left(\begin{array}{c}
j \\
j-l
\end{array}\right)(-\pi i)^{l} \frac{\partial^{j+1-l}}{\partial \bar{z}^{j+1-l}} q(z, \bar{z}) \\
= & i(-\pi i) e^{-\pi i(z+\bar{z})}\left(\begin{array}{c}
j \\
0
\end{array}\right)(-\pi i)^{j} q(z, \bar{z}) \\
& +i(-\pi i) e^{-\pi i(z+\bar{z})} \sum_{l=0}^{j-1}\left(\begin{array}{c}
j \\
j-l
\end{array}\right)(-\pi i)^{l} \frac{\partial^{j-l}}{\partial \bar{z}^{j-l}} q(z, \bar{z}) \\
& +i e^{-\pi i(z+\bar{z})} \sum_{l=1}^{j}\left(\begin{array}{c}
j \\
j-l
\end{array}\right)(-\pi i)^{l} \frac{\partial^{j+1-l}}{\partial \bar{z}^{j+1-l}} q(z, \bar{z}) \\
& +i e^{-\pi i(z+\bar{z})}\left(\begin{array}{l}
j \\
j
\end{array}\right) \frac{\partial^{j+1}}{\partial \bar{z}^{j+1}} q(z, \bar{z}) .
\end{aligned}
$$


Putting the middle two summations together using the change of summation $k=l+1$ on the first and noting

$$
\left(\begin{array}{c}
j \\
j-l
\end{array}\right)+\left(\begin{array}{c}
j \\
j-l+1
\end{array}\right)=\left(\begin{array}{c}
j+1 \\
j+1-l
\end{array}\right)
$$

verifies the lemma for $r=j+1$. By induction the lemma is proved. Q.E.D.

Using Lemma 4.1 we see by inspection that if $q^{*}$ satisfies the conditions of the lemma and furthermore $\partial^{j} q^{*}(0,0) / \partial \bar{z}^{j}=0$ whenever $j$ satisfies both $0 \leqslant j \leqslant n$ and $j=3 \bmod 4$ we have $\partial^{j} q^{*}(0,0) / \partial \bar{z}^{j}=0$ for $0 \leqslant j \leqslant n$.

LEMMA 4.2. For $q^{*} \in \mathcal{C}^{n}\left(\Pi^{2}\right), n \geqslant 1$, satisfying (4.3) and $\partial^{j} q^{*}(0,0) / \partial \bar{z}^{j}=0$ whenever $j$ satisfies $0 \leqslant j<n$ and $j \equiv 3 \bmod 4$, we have $q^{*}(z, \bar{z}) / z \in e^{n-1}$.

Proof. Except at $z=0$ it is obvious that $q^{*}(z, \bar{z}) / z$ is of class $e^{n-1}$. Write $q^{*}(z, \bar{z})=s(z, \bar{z})+R(z, \bar{z})$ where $s(z, \bar{z})$ is a polynomial in $z, \bar{z}$ of total degree $<n$ and $R(z, z)$ has all its partials of degree $<n$ vanishing at $(0,0)$. Since $\partial^{j} q^{*}(0,0) / \partial \bar{z}^{j}=0,0 \leqslant j \leqslant n$, it follows that $s(z, \bar{z}) / z$ is a polynomial in $z, \bar{z}$. Hence we must show $R(z, \bar{z}) / z \in \mathrm{C}^{n-1}$. Let $j+k<n$ and consider

$$
S(z, \bar{z})=\frac{\partial^{j+k}}{\partial z^{j} \partial \bar{z}^{-k}} \frac{R(z, \bar{z})}{z} .
$$

Using Taylor's theorem on each of the partials occurring in the numerator of $S$ along with the vanishing of all the partials of $R(z, z)$ of degree $\leqslant n$ at $(0,0)$ we can write $S(z, \bar{z})$ as the sum of products $z \bar{z}^{m} / z^{2^{j}}, l+m=2^{j}$, times partials of $R(z, \bar{z})$ of degree $j+k+1$ evaluated at points which tend to $(0,0)$ as $(z, \bar{z})$ tends to $(0,0)$. But $z^{l} \bar{z}^{m} / z^{2^{j}}$ is bounded near $(0,0)$ and the $(j+k+1)$ th partials of $R(z, \bar{z})$ all vanish at $(0,0)$. Hence the lemma is proved. Q.E.D.

We can now prove the eigenvalue one space version of Theorem 3. Write $F=p_{1} \psi+q_{1} \psi^{*}$ with $p_{1}, q_{1} \in \mathcal{C}^{n}\left(\Pi^{2}\right)$ satisfying (3.1) and (3.2). Observe that $\gamma_{1}^{3} \beta_{-i}$ has as its leading term in its Taylor series at $\left(\frac{1}{2}, \frac{1}{2}\right)$ some constant times $\bar{z}^{-3}$. Moreover $\gamma_{1}^{3} \beta_{-i}$ satisfies (3.2). Hence

$$
\left(q_{1}-a_{0} \gamma_{1}^{3} \beta_{-i}\right) \psi^{*} \in \mathcal{C}_{1}^{n} \cap Y_{1} \text { and } \frac{\partial^{3}}{\partial \bar{z}^{3}}\left(q_{1}-a_{0} \gamma_{1}^{3} \beta_{-i}\right)\left(\frac{1}{2}, \frac{1}{2}\right)=0
$$

for some $a_{0} \in \mathrm{C}$. We continue this process until we have a function

$$
q=q_{1}-\sum_{0<4 l+3<n} a_{l} \gamma_{1}^{4 l+3} \beta_{-i}, \quad a_{l} \in \mathbf{C},
$$

such that $\partial^{j} q\left(\frac{1}{2}, \frac{1}{2}\right) / \partial \bar{z}^{j}=0$ whenever both $0 \leqslant j \leqslant n$ and $j \equiv 3 \bmod 4$ and $q \psi^{*} \in \mathcal{C}_{1}^{n} \cap Y_{1}$. By the preceding lemma we can then write

$$
F=\left(p_{1}+q \psi^{*} / \psi\right) \psi+h \psi_{-i}^{*}
$$

where $p=p_{1}+q \psi^{*} / \psi \in \mathrm{C}^{n-1}$ and $h=\sum_{0<4 l+3<n} a_{l} \gamma_{1}^{4 l+3}$. 
It remains to prove uniqueness. Suppose $p \psi=h \psi_{-i}^{*} \in \mathcal{C}_{1}^{n} \cap Y_{1}$ where $p \in \mathrm{e}^{n-1}$ and $h=\Sigma_{0<4 l+3<n} a_{l} \gamma_{1}^{4 l+3}$. Then $p=h \psi_{-i}^{*} / \psi \in \mathfrak{C}^{n-1}$ and since $\psi_{-i}^{*}$ does not vanish near $\left(\frac{1}{2}, \frac{1}{2}\right)$ we have $h / \psi \in \mathcal{C}^{n-1}$. This can occur only if $h \equiv 0$ and uniqueness is verified.

The analysis of the other eigenvalue spaces proceeds in exactly the same way. Hence we have proved Theorem 3.1. The nature of the representation for functions in the eigenvalue spaces is given in the following corollary. The details are trivial and left to the reader.

Corollary 4.1. Let $F \in \mathcal{C}_{1}^{n}$ and write $F=p \cdot \psi+h \psi_{-i}^{*}$ where $p \in$ $e^{n-1}\left(\Pi^{2}\right)$ and $h=\sum_{0<1<n} a_{l} \gamma_{1}^{l}$. Then

(1) If $F \in Y_{1}, h=\sum a_{4 l+3} \gamma_{1}^{4 l+3}$.

(2) If $F \in Y_{-1}, h=\sum a_{4 l+1} \gamma_{1}^{4 l+1}$.

(3) If $F \in Y_{i}, h=\sum a_{4 l+2} \gamma_{1}^{4 l+2}$.

(4) If $F \in Y_{-i}, h=\Sigma a_{4 l} \gamma_{1}^{4 l}$.

We should also point out before we leave this section that the same techniques can be used to prove the following theorem and corollary.

THEOREM 4.2. Let $F \in \mathfrak{C}_{1}^{n}$. Then there exists 'a unique polynomial $k=$ $\sum_{l=0}^{n} a_{l} \gamma_{2}^{l}$ in $\gamma_{2}=\sin x+i \sin y$ and a unique $q \in \mathcal{C}^{n-1}\left(\Pi^{2}\right)$ such that $F=k$. $\psi+q \psi^{*}$.

CoRollary 4.2. Writing $F=k \cdot \psi+q \psi^{*}$ as in Theorem 4.2 we have

(1) $F \in Y_{1}$ implies $k=\sum a_{41} \gamma_{2}^{4 l}$,

(2) $F \in Y_{-1}$ implies $k=\sum a_{4 l+2} \gamma_{2}^{4 l+2}$,

(3) $F \in Y_{i}$ implies $k=\sum a_{4 l+3} \gamma_{2}^{4 l+3}$,

(4) $F \in Y_{-i}$ implies $k=\sum a_{4 l+1} \gamma_{2}^{4 l+1}$.

5. Fourier analysis. We begin our discussion by explicitly computing for the decomposition $F=p \cdot \psi+\left(\Sigma_{0}^{3} a_{j} \gamma_{1}^{j}\right) \psi_{-i}^{*}$ where $F \in \mathcal{C}_{1}^{3}, p \in \mathcal{C}^{2}\left(\Pi^{2}\right)$ the coefficients $a_{j}, j=0,1,2,3$. Note that the Taylor expansion of $\gamma_{1}$ at $\left(\frac{1}{2}, \frac{1}{2}\right)$ is $-2\left[\left(x-\frac{1}{2}\right)-i\left(y-\frac{1}{2}\right)\right]+$ higher order terms and $\beta_{-i}\left(\frac{1}{2}, \frac{1}{2}\right)=4$. Also it is easy to see that $\psi^{*}\left(\frac{1}{2}, \frac{1}{2}, 0\right)=-\theta[0](0 \mid i)$.

LEMMA 5.1. For $F \in \mathfrak{C}_{1}^{3}$ given by $F=p \psi+\left(\sum_{j=0}^{3} a_{j} \gamma_{1}^{j}\right) \psi_{-i}^{*}$ with $p \in \mathcal{C}^{2}\left(\Pi^{2}\right)$ we have

$$
a_{j}=\frac{(-1)^{j+1}}{4 \cdot 2^{j}} \frac{\partial^{j} / \partial \bar{z}^{j} F\left(\frac{1}{2}, \frac{1}{2}, 0\right)}{\theta\left[\begin{array}{l}
0 \\
0
\end{array}\right](0 \mid i)}, \quad j=0,1,2,3 .
$$

Proof. For simplicity assume $F \in Y_{1}$ and then $F=p \psi+a_{3} \gamma_{1}^{3} \psi_{-i}^{*}$. Since $\partial^{3}(p \psi)\left(\frac{1}{2}, \frac{1}{2}, 0\right) / \partial \bar{z}=0$ the result follows from the preceding discussion. The other cases are handled similarly. 
Take $F=p \psi+\left(\Sigma_{0}^{3} a_{j} \gamma_{1}^{j}\right) \psi_{-i}^{*} \in \mathcal{C}_{1}^{3}$ where $p \in \mathcal{C}^{2}\left(\Pi^{2}\right)$. Since $p$ is the uniform limit of its associated Fourier series, $p=\Sigma b_{j k} e^{2 \pi i(j x+k y)}$, we can write

$$
F=\Sigma b_{j k} \psi\langle j, k\rangle+\sum_{|l|+|m|<3} c_{l m} \psi_{-i}^{*}\langle l, m\rangle
$$

where

$$
\psi\langle j, k\rangle=\chi_{j k} \psi, \quad \psi_{-i}^{*}\langle j, k\rangle=\chi_{j k} \psi_{-i}^{*} \quad \text { and } \quad \chi_{j k}=e^{2 \pi i(j x+k y)} .
$$

We call (5.1) the $\mathcal{C}^{2}$-expansion of $F$. Hence we have associated in a unique fashion to each $F \in C_{1}^{3}$ its $\bigodot^{2}$-expansion and using Lemma 5.1 the coefficients are determinable.

As a final remark we note that the functions $\{\psi\langle j, k\rangle\}, j, k \in Z$ define an $\mathfrak{L}^{2}$-basis of $H_{1}$ in the sense that they are dense in the $\mathfrak{L}^{2}$-norm and no smaller subset is dense (see [2]). The crucial point of (5.1) is that except for the computable error given by the last series on the right this $\mathfrak{L}^{2}$-basis can be employed to generalize the classicial theorem stated in the introduction.

CoRollary 5.1.1. For $F \in \mathcal{C}_{1}^{3}$ satisfying $\partial^{j} / \partial \bar{z}^{j} F\left(\frac{1}{2}, \frac{1}{2}, 0\right)=0, j=$ $0,1,2,3$, we have $F=\Sigma b_{j k} \psi\langle j, k\rangle$, the series absolutely and uniformly converging.

6. Analysis in $\mathscr{L}^{2}\left(\Gamma \backslash N_{3}\right)$. The first step in our investigation of $\mathfrak{L}^{2}\left(\Gamma \backslash N_{3}\right)$ is the extension of our main theorem to the spaces $H_{n}, n \neq 0,1$. Since complex conjugation identifies $H_{n}$ and $H_{-n}, n \neq 0$, it suffices to restrict our attention to $H_{n}, n \geqslant 1$. The procedure is to recall without proof from [2] the necessary algebraic machinery which will allow for the translation of our results from $H_{1}$ to $H_{n}$.

Let $\tau_{n}=\left(\begin{array}{cc}1 & 0 \\ 0\end{array}\right)$ and denote by $\tau_{n}$ as well the corresponding automorphism of $N_{3}$ given by

$$
\tau_{n}(x, y, z)=(n x, y, n z), \quad(x, y, z) \in N_{3} .
$$

Since $\tau_{n}(\Gamma) \subset \Gamma$ the map of $\mathcal{L}^{2}\left(\Gamma \backslash N_{3}\right)$ given by

$$
\left(\mathcal{Q}_{n} F\right)(x, y, z)=F(n x, y, n z), \quad F \in \mathcal{L}^{2}\left(\Gamma \backslash N_{3}\right)
$$

is an isometry of $\mathcal{L}^{2}\left(\Gamma \backslash N_{3}\right)$. It is shown in [2] that for $n>1, \mathcal{Q}_{n}\left(H_{1}\right)$ is an $R$-invariant and irreducible subspace of $H_{n}$.

Let $\Lambda_{n}=\{(a / n, b / n, z): a, b \in Z, z \in R\}, n \geqslant 1$. Clearly $\Lambda_{n}$, which is called the left stabilizer of $H_{n}$, is a subgroup of $N_{3}$. We can define a unitary representation $L_{n}$ of $\Lambda_{n}$ on $H_{n}$ by the formula

$$
L_{n}(g) F(h)=F\left(g^{-1} h\right), \quad g \in \Lambda_{n}, h \in N_{3}, F \in H_{n} .
$$

This construction first appears in [1] and later in [2]. In either case it is verified that for $n \geqslant 1, H_{n}=\sum_{j=0}^{n-1} \oplus L_{n}(0,-j / n, 0)\left(\vartheta_{n} H_{1}\right)$. Set 


$$
\begin{aligned}
H_{n j} & =L_{n}(0,-j / n, 0)\left(\tau_{n} H_{1}\right), \quad N>1, j=0, \ldots, n-1, \\
\psi_{n j} & =L_{n}(0,-j / n, 0)\left(\bigcup_{n} \psi\right), \quad n \geqslant 1, j=0, \ldots, n-1, \\
\psi_{n j}^{*} & =L_{n}(0,-j / n, 0)\left(\bigcup_{n} \psi^{*}\right), \quad n \geqslant 1, j=0, \ldots, n-1, \\
\psi_{-i, n j}^{*} & =L_{n}(0,-j / n, 0)\left(\bigcup_{n} \psi_{-i}^{*}\right), \quad n \geqslant 1, j=0, \ldots, n-1 .
\end{aligned}
$$

The final preliminary remark concerns the projection operators onto the spaces $H_{n}$ and $H_{n j}$. Let $n>1, j=0, \ldots, n-1$, and denote by $p_{n}$, the projection of $\mathscr{L}^{2}\left(\Gamma \backslash N_{3}\right)$ onto $H_{n}$, and by $p_{n j}$, the projection of $H_{n}$ onto $H_{n j}$. It easily follows from the explicit description of $p_{n}$ on page 5 of [2] that $p_{n}\left(e^{r}\left(\Gamma \backslash N_{3}\right)\right)=\bigodot_{n}^{r}, r \geqslant 0$. Also from [2, Theorem II.8] we have $p_{n j}\left(\bigodot_{n}^{r}\right)=\bigodot_{n}^{r}$ $\cap H_{n j}, r \geqslant 0$.

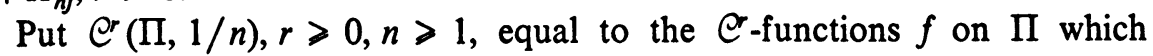
satisfy the additional property $f(x+1 / n, y)=f(x, y)$.

Clearly $C_{n}^{r}$ is a $\operatorname{Cr}(\Pi, 1 / n)$-module. Moreover Lemma 2.2 generalizes to give the following.

LEMmA 6.2. Let $F \in \mathcal{C}_{n}^{r}, r \geqslant 0, n \geqslant 1$. Then there exist functions $g_{j}, h_{j} \in$ $\operatorname{er}\left(\Pi^{2}, 1 / n\right), j=0, \ldots, n-1$, such that

$$
F=\sum_{j=0}^{n-1}\left(g_{j} \psi_{n j}+h_{j} \psi_{n j}^{*}\right)
$$

For a proof see [2, Theorem II.4] or simply consider the above decomposition of $H_{n}$ into the $H_{n j}$ 's. We remark that as in Lemma 2.2 the functions $g_{j}$ and $h_{j}$ are not determined uniquely.

THEOREM 6.2. Let $F \in \mathcal{C}_{n}, r \geqslant 1, n \geqslant 1$. Then there exists a unique set of functions $p_{j} \in \mathrm{C}^{-1}\left(\Pi^{2}, 1 / n\right), j=0, \ldots, n-1$, and a unique set of polynomials $h_{j}$ in $\gamma_{1, n j}=L_{n}(0,-j / n, 0)\left(\vartheta_{n} \gamma_{1}\right), j=0, \ldots, n-1$, such that

$$
F=\sum_{j=0}^{n-1} p_{j} \psi_{n j}+\sum_{j=0}^{n-1} h_{j} \psi_{-i, i j}^{*}
$$

PROOF. Writing $F=\sum_{j=0}^{n-1} F_{j}$ where $F_{j} \in \bigodot_{n}^{r} \cap H_{n j}$, we have

$$
F_{j}=L_{n}(0,-j / n, 0)\left(\mathcal{U}_{n} G_{j}\right) \text { where } G_{j} \in \bigodot_{1} .
$$

By Theorem 4.1, $G_{j}=p_{j} \psi+h_{j} \psi_{-i}^{*}$ where $p_{j} \in \mathrm{C}^{-1}\left(\Pi^{2}\right)$ and $h_{j}$ is a polynomial in $\gamma_{1}=\sin x-i \sin y$. Also the $p_{j}, h_{j}$ are uniquely determined by this requirement. Hence $F_{j}=p_{j}^{\prime} \psi_{n j}+h_{j}^{\prime} \psi_{-i, n j}^{*}$ where $p_{j}^{\prime}=L_{n}(0,-j / n, 0)\left(\vartheta_{n} p_{j}\right)$ and $h_{j}^{\prime}=L_{n}(0,-j / n, 0)\left(\mathcal{Q}_{n} h_{j}\right)$. The theorem follows.

The following lemma permits us to put all the pieces in place. We give a proof for lack of a convenient reference. 
Lemma 6.3. Let $F \in \mathcal{C}\left(\Gamma \backslash N_{3}\right), r \geqslant 1$, and write $F=\Sigma_{n \in Z} F_{n}$ where $F_{n} \in$ $H_{n}, n \in Z$. Then the series $\Sigma_{n \in Z} F_{n}$ converges absolutely and uniformly on $\Gamma \backslash N_{3}$.

PRoof. There is no loss in generality in the assumption that $F=$ $\sum_{n>1} F_{n}, F_{n} \in H_{n}, n \geqslant 1$. Writing $F_{n}=\alpha_{n}(x, y) e^{2 \pi i n z}$ we have

$$
\alpha_{n}(x, y)=\int_{0}^{1} F(x, y, z) e^{-2 \pi i n z} d z .
$$

For fixed $x, y \in[0,1]$ the series $\Sigma_{n>1} F_{n}(x, y, z)$ absolutely converges since $\partial F(x, y, z) / \partial z$ exists and is continuous. In the usual manner differentiating with respect to $z$ gives

$$
2 \pi i n \alpha_{n}(x, y)=\int_{0}^{1} \frac{\partial F}{\partial z}(x, y, z) e^{-2 \pi i n z} d z, \quad n>1 .
$$

By the Plancherel theorem it follows that for all $m \geqslant 1$,

$$
2 \pi \sum_{n>m}|n|^{2}\left|\alpha_{n}(x, y)\right|^{2}<\int_{0}^{1}\left|\frac{\partial F}{\partial z}(x, y, z)\right|^{2} d z .
$$

Hence Hölder's inequality implies that for $m>1$,

$$
2 \pi \sum_{n>m>1}\left|\alpha_{n}(x, y)\right|<\sup _{0<x, y, z<1} \sqrt{2 \pi}\left|\frac{\partial F}{\partial z}(x, y, z)\right|\left(\sum_{n>m>1}|n|^{-2}\right)^{\frac{1}{2}} .
$$

The theorem clearly follows.

Our final theorem is a simple consequence of the previous lemma and theorem.

THEOREM 6.4. For $F \in \mathcal{C}\left(\Gamma \backslash N_{3}\right), r>1$, there are uniquely determined functions $p_{n j} \in \mathcal{C}^{r-1}(\Pi, 1 /|n|)$ and polynomials $h_{n j}$ in

$$
\gamma_{1, n j}=\sin n x-i(\sin (y+j / n))
$$

where $n \neq 0, j=0,1, \ldots,|n|-1$, such that

$$
F=F_{0}+\sum_{n>1}\left[\sum_{j=0}^{n-1}\left(p_{n j} \psi_{n j}+\bar{p}_{-n j} \bar{\psi}_{n j}\right)\right]+\sum_{n>1}\left[\sum_{j=0}^{n-1}\left(h_{n j} \psi_{-i, n j}^{*}+\bar{h}_{-n j} \bar{\psi}_{-i, n j}^{*}\right)\right]
$$

the series uniformly converging with respect to $n$.

\section{REFERENCES}

1. L. Auslander and J. Brezin, Translation-invariant subspaces in $L^{2}$ of a compact nilmanifold. I, Invent. Math. 20 (1973), 1-14. MR 48 \#464. 
2. L. Auslander and R. Tolimieri (with H. Rauch), Abelian harmonic analysis, theta functions and harmonic analysis on a nilmanifold, Lecture Notes in Math., vol. 436, Springer-Verlag, Berlin and New York, 1975.

3. J. Brezin, Function theory on metabelian solvmanifolds, J. Functional Analysis 10 (1972), 33-51. MR 50 \#899.

4. Harmonic analysis on nilmanifolds, Trans. Amer. Math. Soc. 150 (1970), 611-618. MR 43 \#4967.

5. A. Weil, Sur certains groupes d'opérateurs unitaires, Acta Math. 111 (1964), 143-211. MR 29 \#2324.

Department of Mathematics, University of Connecticut, Storrs, Connecticut 06268 